ESAIM: PROCEEDINGS AND SURVEYS, November 2014, Vol. 46, p. 111-124

ECIT 2012 - Witold Jarczyk, Daniele Fournier-Prunaret, João Manuel Gonçalves Cabral

\title{
SYNCHRONIZATION AND INFORMATION TRANSMISSION IN NETWORKS
}

\author{
ACIlina CAneCO ${ }^{1}$ And J. LeOnel Rocha ${ }^{2}$
}

\begin{abstract}
The amount of information produced by a network may be measured by the mutual information rate. This measure, the Kolmogorov-Sinai entropy and the synchronization interval are expressed in terms of the transversal Lyapunov exponents. Thus, these concepts are related and we proved that the larger the synchronization is, the larger the rate with which information is exchanged between nodes in the network. In fact, as the coupling parameter increases, the mutual information rate increases to a maximum at the synchronization interval and then decreases. Moreover, the KolmogorovSinai entropy decreases until reaching a minimum at the synchronization interval and then increases. We present some numerical simulations considering two different versions of coupling two maps, a complete network and a lattice, which confirmed our theoretical results.
\end{abstract}

AMS (2010) subject classification. 34D06; 94A15; 28D20; 05C82; $03 \mathrm{G} 10$.

Keywords. Synchronization, information theory, Lyapunov exponents, mutual information rate, Kolmogorov-Sinai entropy, complex networks.

Résumé. La quantité d'information produite par le réseau, peut être mesurée par la taux mutuelle d'information. Cette mesure, l'entropie de Kolmogorov-Sinai et l'intervalle de synchronisation sont exprimés en termes des exposants de Lyapunov transversales. Ainsi, ces concepts sont liés et nous avons prouvé que plus la synchronisation est élevée, plus grand est la vitesse à laquelle l'information est échangée entre les noeuds du réseau. En fait, si le paramètre de couplage augmente, le taux mutuelle d'information augmente jusqu'à un maximum, à l'intervalle de synchronisation et, diminue ensuite. En outre, l'entropie de Kolmogorov-Sinai diminue jusqu'à atteindre un minimum, à l'intervalle de synchronisation et, ensuite elle augmente. Nous présentons aussi, quelques simulations numériques en considérant deux versions diferentes de coupler deux systèmes, un réseau complet et un treillis, qui ont confirmé nos résultats théoriques.

Mots clefs. Synchronisation, theorie d'information, exposants de Lyapunov, taux mutuelle d'information, entropie de Kolmogorov-Sinai, réseau complexes.

\section{INTRODUCTION AND MOTIVATION}

Information theory is a branch of mathematics and engineering concerning the quantification of information and it benefits of matters like mathematics, statistics, computer science, physics, neurobiology and electrical engineering. Its applications range from neurobiology and others communication networks, cryptography, language processing, evolution of molecular codes, model selection in ecology, quantum computing and many others

\footnotetext{
${ }^{1}$ Instituto Superior de Engenharia de Lisboa - ISEL, ADM and CIMA-UE, Rua Conselheiro Emdio Navarro, 1, 1959-007 Lisboa, Portugal, acilina@adm.isel.pt

${ }^{2}$ Instituto Superior de Engenharia de Lisboa - ISEL, ADM and CEAUL, Rua Conselheiro Emdio Navarro, 1, 1959-007 Lisboa, Portugal,jrocha@adm.isel.pt
}

(C) EDP Sciences, SMAI 2014 
forms of data analysis. Its impact has been of fundamental importance to the success of missions into the deep space, the conception of compact disc, the mobile phones, the development of Internet, the study of human perception and of linguistics and many other fields.

The communication between cells is one of the most important characteristics of all human beings and animals, since it allows the various components of such complex systems to work together. Among the various types of intercellular communication, there is communication between neurons, the brain cells, that exchange information via chemical and electrical synapses. It is believed that the behavior is related to the way the neurons are connected (the topology of the network), while the perception is linked to synchronization. This hypothesis comes from the binding hypothesis, see [3] and references therein, which claims that the synchronization functionally binds neural networks that encode the same feature. This hypothesis raised one of the most important debates in contemporary neurobiology because desynchronization also seems to play an important role in perception. Despite the explosive growth in the field of complex networks, it is still unclear under what conditions the synchronization promotes the transmission of information, and which topology facilitates the information flow.

In this work we study the complex relationship between synchronization and transmission of information. We are mainly interested in the relationship between the rate of information that can be transmitted from one point to another in an active network, considered as the mutual information rate, the level of synchronization between the elements and the topology of the network. In Sec.2, we present some concepts to be used in the following, such as, the communication channel, the channel capacity, the network capacity, the synchronization interval, the Kolmogorov-Sinai entropy, the mutual information rate and conditions for complete synchronization.

Sec.3 is devoted to the main result of the paper, Theorem 3.1. In general conditions, we found that, as the coupling parameter increases from zero, the Kolmogorov-Sinai entropy decreases until reaching a minimum at the synchronization interval and then increases. The mutual information rate behaves in the opposite way, it increases to a maximum and then decreases. For values of the coupling parameter for which there is complete synchronization, the Kolmogorov-Sinai entropy is equal to the mutual information rate. As the coupling parameter increases from zero, the level of synchronization increases and this implies that the flux of information, measured by the mutual information rate, increases also so, the larger the synchronization is the larger the rate with which information is exchanged between nodes in the network, the so called mutual information rate.

In Sec.4, numerical simulations are presented, where we consider several types of networks and we observe the relationship between the level of synchronization and the evolution of the mutual information rate and the Kolmogorov-Sinai entropy. In the nodes of all types of networks we consider the same dynamical system, for example, the skew-tent map studied by Hasler and Maistrenko, [7]. In Subsecs.4.1 and 4.2 it is analyzed the coupling of two of these skew-tent maps, version 1 and 2, respectively. In Subsecs.4.3 and 4.4 it is studied a complete network and a lattice, respectively. In all these types of network topologies, we determine the synchronization region as a function of the coupling parameter and the skew-tent map parameter. We also evaluate the Kolmogorov-Sinai entropy and the mutual information rate.

\section{Preliminaries}

A communication channel represents a pathway through which information is exchanged. In this work, a communication channel is considered to be formed by a pair of elements. One element represents a transmitter $S_{i}$ and the other a receiver $S_{j}$, where the information about the transmitter can be measured, see [1] and [2]. In a network, each one of the links between them, i.e., each one of the edges of the corresponding graph, represents a communication channel. If the network with $N$ nodes is complete, i.e., it has a all-to-all coupling, it has $\frac{N(N-1)}{2}$ edges or communication channels, but they are all one connection apart from each other.

In [1], it is defined the mutual information rate (MIR) between one transmitter $S_{i}$ and one receiver $S_{j}$, denoted by $I_{C}\left(S_{i}, S_{j}\right)$, by

$$
I_{C}\left(S_{i}, S_{j}\right)=\sum \lambda_{\|}^{+}-\sum \lambda_{\perp}^{+}
$$


where $\lambda_{\|}^{+}$denotes the positive Lyapunov exponents associated to the synchronization manifold and $\lambda_{\perp}^{+}$denotes the positive Lyapunov exponents associated to the transversal manifold, between $S_{i}$ and $S_{j}$. $\sum \lambda_{\|}^{+}$represents the information (entropy production per time unit) produced by the synchronous trajectories and corresponds to the amount of information transmitted. On the other hand, $\sum \lambda_{\perp}^{+}$represents the information produced by the nonsynchronous trajectories and corresponds to the information lost in the transmission, the information that is erroneously retrieved in the receiver. For more details and references see for example [1], [2] and [3].

The Kolmogorov-Sinai entropy, denoted by $H_{K S}$, quantifies the amount of uncertainty that defines information. For systems with a measurable (the trajectory is bounded to a finite domain) and ergodic (average quantities can be calculated in space and time) invariant (with respect to time translations of the system and to smooth transformations) natural measure, that is smooth along the unstable manifold, $H_{K S}$ equals the sum of the positive Lyapunov exponents, i.e.,

$$
H_{K S}=\sum \lambda^{+}
$$

where $\lambda^{+}$represents all the positive Lyapunov exponents of the network, see for example [8], [9] and [12].

The channel capacity is defined in terms of the proposed upper bound for the mutual information rate. It measures the local maximal rate of information that two elements in a given network are able to exchange, a point-to-point measure of information exchange, [2]. The capacity of a chaotic channel, between systems $S_{i}$ and $S_{j}$, as defined in Shannon work, see for example [13], denoted by $C\left(S_{i}, S_{j}\right)$, is the maximum of the mutual information rate, i.e.,

$$
C\left(S_{i}, S_{j}\right)=\max \left[I_{C}\left(S_{i}, S_{j}\right)\right]
$$

for all possible values of the coupling parameter, satisfying the condition

$$
C\left(S_{i}, S_{j}\right) \leq H_{K S}
$$

The network capacity is the maximum of the Kolmogorov-Sinai entropy, for many possible network configurations with a given number of elements. It gives the amount of independent information that can be simultaneously transmitted within the whole network, and naturally bounds the value of the MIR in the channels, which concerns only the transmission of information between two elements. It gives the amount of independent information that can be simultaneously transmitted within the whole network. Network capacity bounds the value of the MIR in the channels, which concerns only the transmission of information between two elements. While the channel capacity is bounded and does not depend on the number of elements forming the network, the network capacity depends on the number of elements forming the network.

Consider a network of $N$ identical chaotic dynamical oscillators, described by a connected and unoriented graph, with no loops and no multiple edges. In each node the dynamics of the oscillators is defined by $\dot{x}_{i}=f\left(x_{i}\right)$, with $f: \mathbb{R}^{n} \rightarrow \mathbb{R}^{n}$ and $x_{i} \in \mathbb{R}^{n}$ is the state variables of the node $i$. The state equations of this network are

$$
\dot{x}_{i}=f\left(x_{i}\right)+\sigma \sum_{j=1}^{N} l_{i j} x_{j}, \text { with } i=1,2, \ldots, N
$$

where $\sigma>0$ is the coupling parameter, $L=\left[l_{i j}\right]=D-A$ is the Laplacian matrix or coupling configuration of the network. The network Eq.(5) achieves asymptotical synchronization if $x_{1}(t)=x_{2}(t)=\ldots=x_{N}(t) \underset{t \rightarrow \infty}{\rightarrow} e(t)$, where $e(t)$ is a solution of an isolated node (equilibrium point, periodic orbit or chaotic attractor), satisfying $\dot{e}(t)=f(e(t))$.

A network of this type, having in each node identical piecewise linear expanding maps $f$, synchronizes in the following interval

$$
\sigma_{1}=\frac{1-e^{-\chi_{\mu_{1}}(f)}}{\lambda_{2}}<\sigma<\frac{1+e^{-\chi_{\mu_{1}}(f)}}{\lambda_{N}}=\sigma_{2}
$$

where $0=\lambda_{1}<\lambda_{2} \leq \ldots \leq \lambda_{N}$ are the eigenvalues of the coupling matrix $L$ and $\chi_{\mu_{1}}(f)$ are the Lyapunov exponent of $f$, see [5]. We denote by $] \sigma_{1}, \sigma_{2}$ [ the synchronization interval. 
One of the most popular and widely used criterion to study the stability of the synchronized motion is the use of the Lyapunov exponents as average measurements of expansion or shrinkage of small displacements along the synchronized trajectory. The stability problem of identical coupled systems can be formulated in a very general way by addressing the question of the stability of the synchronization manifold $x \equiv y$, or equivalently by studying the temporal evolution of the synchronization error $e \equiv y-x$, where $x$ and $y$ are the state vectors of the coupled systems, [4]. The evolution of the synchronization error $e$ is given by

$$
\dot{e}=f(x ; s(t))-f(y ; s(t)) \text {, }
$$

where $t$ is the time, $f$ is the vector field such that $\dot{x}=f(x)$ governs the dynamics in each node of the network and $s(t)$ denotes a trajectory on the synchronization manifold.

A complete synchronization regime exists when the synchronization manifold is asymptotically stable, for all possible trajectories $s(t)$ of the system within the chaotic attractor, [4]. This property can be proved by using stability analysis of the linearized system for small $e$, i.e.,

$$
\dot{e}=D_{x}(s(t)) e,
$$

where $D_{x}$ is the Jacobian of the vector field $f$, evaluated onto the trajectory $s(t)$.

Normally, when the trajectory $s(t)$ is constant (fixed point) or periodic (limit cycle), the study of the stability problem can be made by means of evaluating the eigenvalues of $D_{x}$ or the Floquet multipliers, [15]. However, if the response system is driven by a chaotic signal, this method will not work. A possible solution is calculating the Lyapunov exponents of system (8), [10] and [11]. These exponents are usually called conditional Lyapunov exponents because they are the Lyapunov exponents of the response system under the explicit constrain that they must be calculated on the trajectory $s(t)$. Alternatively, they are called transversal Lyapunov exponents because they correspond to directions which are transverse to the synchronization manifold $x \equiv y$. Basically, two coupled chaotic systems have two sets of conditional exponents. One set is associated with the synchronization manifold and the other one associated with the transversal manifold.

In [10] and [11] it was establish that complete synchronization (CS) can be achieved provided that all the conditional Lyapunov exponents are negative. Since then, some authors have reported theirs computational experiments showing that apparently it is possible to achieve synchronization without the negativity of all conditional Lyapunov exponents, [14], and some others have reported that sometimes there is brief lack of synchronization in the region where all the conditional Lyapunov exponents are negative, see [6] and references therein. The negativity of the conditional Lyapunov exponents is a necessary condition for the stability of the synchronized state, [4]. Is also a mathematical expression of the decreasing to zero of the logarithm average of the distance of the solutions on the transverse manifold to the solutions on the synchronization manifold.

\section{Main RESUlts: Synchronization EnhanCES INFOrmation FlOW}

Using the previous concepts, which express quantities such as the mutual information rate, the KolmogorovSinai entropy and the synchronization interval, in terms of the Lyapunov exponents, we can relate the amount of information that flows in a network, with the synchronization level of it. We will present a result, for a network with $N$ vertices, and $n$ different transversal Lyapunov exponents $\lambda_{\perp}$. As the transversal Lyapunov exponents represent the information produced by the nonsynchronous trajectories and corresponds to the information lost in the transmission, having $n$ different transversal Lyapunov exponents $\lambda_{\perp}$ means that we have $n$ different possible values for the mutual information rate $I_{C_{k}}\left(S_{i}, S_{j}\right)$, with $k=1, \ldots, n$. In a network with only two nodes, see Subsecs.4.1 and 4.2, there are just two Lyapunov exponents, one is the $\lambda_{\|}$, associated to the synchronization manifold and the other is the $\lambda_{\perp}$, associated to the transversal manifold. In a complete network, see Subsec.4.3, where each node is connected with all the others nodes, each node is only one connection apart from any of the others, there is just one single transversal Lyapunov exponents $\lambda_{\perp}$. But, in a lattice, such as the example in Subsec.4.4, there are two different transversal Lyapunov exponents $\lambda_{\perp}$, then there are two different types of 
mutual information rate, which correspond to the fact that there are some nodes that are one connection apart and there are other nodes that are two connections apart. So, in the examples of Subsecs.4.1, 4.2 and 4.3, we have $n=1$ and in Subsec. 4.4 we have $n=2$.

Theorem 3.1. Consider a network defined by Eq.(5), with $n \in \mathbb{N}$ distinct transversal Lyapunov exponent $\lambda_{\perp_{k}}$, with $k=1, \ldots, n$. Lets $\sigma \in \mathbb{R}_{0}^{+}$be the coupling parameter and $] \sigma_{1}, \sigma_{2}$ [ be the synchronization interval.

(i) If $\sigma \in\left[0, \sigma_{1}\right]$, then the MIR, $I_{C_{k}}\left(S_{i}, S_{j}\right)$, increases $\forall k=1, \ldots, n$ and $H_{K S}$ decreases.

(ii) If $\sigma \in] \sigma_{1}, \sigma_{2}\left[\right.$, then the $\operatorname{MIR} I_{C_{k}}\left(S_{i}, S_{j}\right), \forall k=1, \ldots, n$ and $H_{K S}$ are equal, i.e.,

$$
I_{C_{k}}\left(S_{i}, S_{j}\right)=H_{K S}=C\left(S_{i}, S_{j}\right), \forall k=1, \ldots, n
$$

and the capacity of the chaotic channels is attained.

(iii) If $\sigma \geq \sigma_{2}$, then the $M I R, I_{C_{k}}\left(S_{i}, S_{j}\right)$, decreases $\forall k=1, \ldots, n$ and $H_{K S}$ increases.

Proof. If there are $n \in \mathbb{N}$ distinct transversal Lyapunov exponent $\lambda_{\perp_{k}}$, there are $n$ distinct values of the mutual information rate, $I_{C_{k}}\left(S_{i}, S_{j}\right)$, with $k=1, \ldots, n$. According to $(1)$ the $\operatorname{MIR}, I_{C_{k}}\left(S_{i}, S_{j}\right)$, to each $k=1, \ldots, n$, is given by

$$
I_{C_{k}}\left(S_{i}, S_{j}\right)=\left\{\begin{array}{ll}
\lambda_{\|}-\lambda_{\perp_{k}} & , \text { if } \lambda_{\perp_{k}}>0 \\
\lambda_{\|} & , \text {if } \lambda_{\perp_{k}} \leq 0
\end{array} .\right.
$$

If $\sigma=0$, then the nodes of the network are uncoupled. Moreover, as the coupling $\sigma$ increases with $0<\sigma<\sigma_{1}$, the larger is the synchronization level and the value of the positives transversal Lyapunov exponent $\lambda_{\perp_{k}}$ decreases to zero. When the complete synchronization is attained, i.e., $\sigma_{1}<\sigma<\sigma_{2}$, all transversal Lyapunov exponent $\lambda_{\perp_{k}}$, with $k=1, \ldots, n$, become negative. So, by Eq.(9) the family of MIR $I_{C_{k}}\left(S_{i}, S_{j}\right)$ increases as the coupling $\sigma$ increases in the interval $\left[0, \sigma_{1}\right]$, and consequently, the amount of information retrieved in the receiver increases, see [2] and [3].

On the other hand, according to (2), the Kolmogorov-Sinai entropy $H_{K S}$ is given by

$$
H_{K S}=\left\{\begin{array}{ll}
\lambda_{\|}+\sum_{k=1}^{n} \lambda_{\perp_{k}} & , \text { if } \lambda_{\perp_{k}}>0 \\
\lambda_{\|} & , \text {if } \lambda_{\perp_{k}} \leq 0
\end{array} .\right.
$$

If the coupling parameter $\sigma \in\left[0, \sigma_{1}\right]$, then there is at most $n$ transversal Lyapunov exponent $\lambda_{\perp_{k}}>0$. As the coupling parameter increases, the level of synchronization increases and the value of the positive transversal Lyapunov exponent decreases to zero, and some of them become negative. So, attending to Eq.(10), the Kolmogorov-Sinai entropy $H_{K S}$ decreases. The first claim is proved.

In the synchronization interval one has $\lambda_{\perp_{k}} \leq 0$, with $k=1, \ldots, n$. So, by Eqs.(9) and (10), we get

$$
I_{C_{k}}\left(S_{i}, S_{j}\right)=H_{K S}, \forall k=1, \ldots, n
$$

i.e., the mutual information rate $I_{C_{k}}$ and the Kolmogorov-Sinai entropy $H_{K S}$ are equal. On the other hand, the maximum of the mutual information rate $I_{C_{k}}$, i.e., the capacity of a chaotic channel, given by (3), is attained, which proves the second claim.

If the coupling parameter $\sigma \geq \sigma_{2}$, then there is at most $n$ transversal Lyapunov exponent $\lambda_{\perp_{k}}>0$, which increases, as the coupling parameter increases. Therefore, attending to Eq.(9), the MIR $I_{C_{k}}\left(S_{i}, S_{j}\right)$, to each $k=1, \ldots, n$, decreases. Similarly, by Eq.(10), the Kolmogorov-Sinai entropy $H_{K S}$ increases. So, the claim (iii) is proved.

We have proved that as the network becomes more synchronized, i.e., the level of synchronization increases, the flux of information, measured by the mutual information rate, increases, attaining its maximum (the channel capacity) in the synchronization interval. 


\section{Numerical SIMUlations}

In this section we present some numerical simulations that confirm the previous results. In all examples we consider networks having in each node the same dynamical system, for instance, a skew tent map depending on a parameter.

\subsection{Coupling of two tent maps: version 1}

Consider the skew-tent map $f:[0,1] \rightarrow[0,1]$, defined by

$$
f(x)=\left\{\begin{array}{cl}
\frac{x}{a} & \text { if } 0 \leq x \leq a \\
\frac{1-x}{1-a} & \text { if } a<x \leq 1
\end{array} .\right.
$$

We suppose $0.5 \leq a<1$, but the results are analogous for $0<a \leq 0.5$. In [7], Hasler and Maistrenko studied the synchronization of two of these identical skew-tent maps, coupled in two different ways. The version 1 of the coupling is given by $\left(x_{k+1}, y_{k+1}\right)=F\left(x_{k}, y_{k}\right)$, with

$$
\left\{\begin{array}{l}
x_{k+1}=f\left(x_{k}\right)+\delta\left(y_{k}-x_{k}\right) \\
y_{k+1}=f\left(y_{k}\right)+\varepsilon\left(x_{k}-y_{k}\right)
\end{array}\right.
$$

where $\delta$ and $\varepsilon$ are two coupling parameters. For points on the synchronization manifold $S \equiv\left\{(x, y) \in \mathbb{R}^{2}: x=y\right\}$, the Jacobian matrix is

$$
D F\left[\begin{array}{l}
x \\
x
\end{array}\right]=\left[\begin{array}{cc}
c-\delta & \delta \\
\varepsilon & c-\varepsilon
\end{array}\right], \text { with } c=c(x)=\left\{\begin{array}{cc}
\frac{1}{a} & \text { if } x \leq a \\
-\frac{1}{1-a} & \text { if } x>a
\end{array} .\right.
$$

The eigenvalues of the Jacobian $D F$ are $\mu_{1}(x)=c$ and $\mu_{2}(x)=c-\varepsilon-\delta$ and the eigenvectors are $v_{1}=(1,1)$ and $v_{2}=(\delta,-\varepsilon)$, respectively. The first eigenvector lies in the subspace $S$, so the correspondent Lyapunov exponent is denoted by $\lambda_{\|}$. The second eigenvector is transversal to $S$ and the correspondent Lyapunov exponent is denoted by $\lambda_{\perp}$. Consider the coupling parameter $d=\varepsilon+\delta$, the Lyapunov exponents are given by

$$
\lambda_{\|}=-a \ln a-(1-a) \ln (1-a) \text { and } \lambda_{\perp}=a \ln \left|\frac{1}{a}-d\right|+(1-a) \ln \left|-\frac{1}{1-a}-d\right| .
$$

Note that, the qualitative behavior of the system depends on the sum of the two coupling parameters, $d=\varepsilon+\delta$. In this case, the parameter $d=\varepsilon+\delta$ has the role of the coupling parameter $\sigma$ refered in the network (5) and we will have a synchronization region $d_{1}<\varepsilon+\delta<d_{2}$, see Fig.2, in the role of the synchronization interval $\sigma_{1}<\sigma<\sigma_{2}$.

For synchronization it is necessary to have $\lambda_{\perp}<0$, see for example [10] and [11]. In Fig. 1 we present, in the left, the region where $\lambda_{\perp}<0$, in terms of the sum of the coupling parameters $d=\varepsilon+\delta \in[0,2]$ as a function of the parameter $a \in[0.5,1]$ and, in the right, it is the graph of $\lambda_{\perp}$ in terms of the sum of the coupling parameters $\varepsilon+\delta$. We have $\lambda_{\perp}<0$ if $0.59<\varepsilon+\delta<1.87$, for $a=0.8$. In Fig.2, we may see the region of synchronization in the $(\varepsilon, \delta)$ parameter plane. For $a=0.8$ the version 1 coupled tent maps (12) synchronize if $0.59<\varepsilon+\delta<1.87$.

According to (9) and (10), in the particular case $n=1$, we evaluate the mutual information rate, the MIR $I_{C}\left(S_{i}, S_{j}\right) \equiv I_{C}$, and the Kolmogorov-Sinai entropy $H_{K S}$, which graphics, for $a=0.8$, are displayed in Fig.3. If $0.59<d=\varepsilon+\delta<1.87$ (complete synchronization interval), then $I_{C}=H_{K S}$. If $d<0.59$, then the MIR $I_{C}$ increases and $H_{K S}$ decreases. On the other hand, if $d>1.87$, then the MIR $I_{C}$ decreases and $H_{K S}$ increases. The results of Theorem (3.1) are verified. 

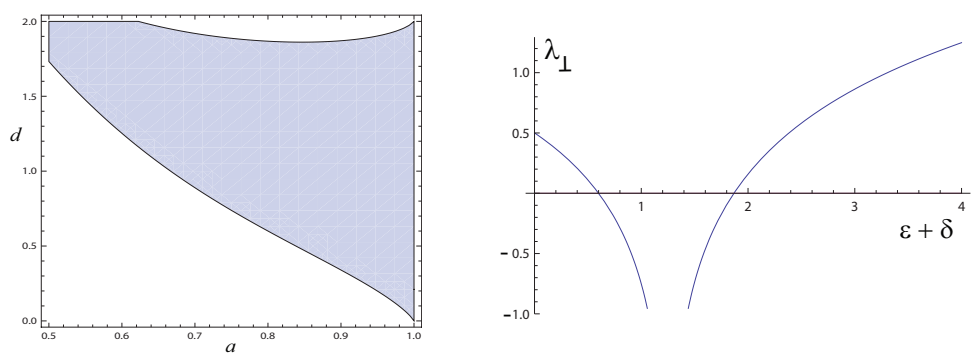

FIgURE 1 . In the left is the region where $\lambda_{\perp}<0$, in terms of the sum of the coupling parameters $d=\varepsilon+\delta \in[0,2]$ as a function of the parameter $a \in[0.5,1]$. In the right, we choose, for instance, $a=0.8$ and we have $\lambda_{\perp}<0$ if $0.59<\varepsilon+\delta<1.87$.

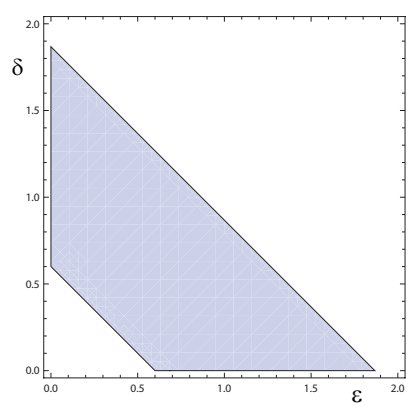

Figure 2. Parameter synchronization region, $0.59<\varepsilon+\delta<1.87$, for $a=0.8$.

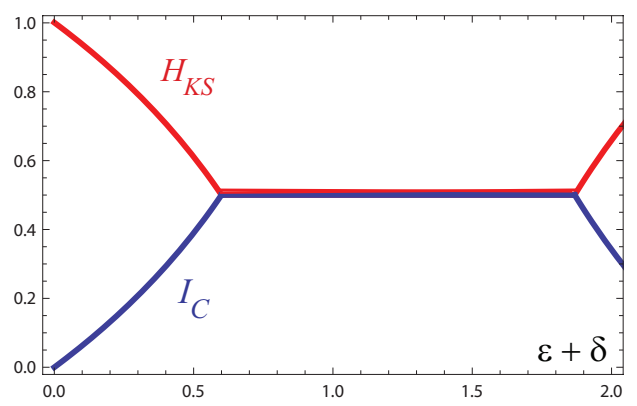

Figure 3. Graphic of $H_{K S}$ (in red) and $I_{C}$ (in blue), for $a=0.8$. If $0.59<d=\varepsilon+\delta<1.87$ (complete synchronization), then $I_{C}=H_{K S}$.

So, with complete synchronization, the errors produced by the nonsynchronous trajectories should vanish and the channel transmits information in its full capacity, while when there is no synchronization, errors may $\operatorname{occur}\left(\lambda_{\perp} \geq 0\right)$.

\subsection{Coupling of two tent maps: version 2}

The version 2 of the coupling is given by $\left(x_{k+1}, y_{k+1}\right)=G\left(x_{k}, y_{k}\right)$, with

$$
\left\{\begin{array}{l}
x_{k+1}=f\left[x_{k}+\delta\left(y_{k}-x_{k}\right)\right] \\
y_{k+1}=f\left[y_{k}+\varepsilon\left(x_{k}-y_{k}\right)\right]
\end{array}\right.
$$


where $f$ is the same skew-tent map (11). For points on the synchronization manifold $S \equiv\left\{(x, y) \in \mathbb{R}^{2}: x=y\right\}$, the Jacobian matrix is given by

$$
D G\left[\begin{array}{l}
x \\
x
\end{array}\right]=c\left[\begin{array}{cc}
1-\delta & \delta \\
\varepsilon & 1-\varepsilon
\end{array}\right]
$$

where $c$ is the slope defined in (13). The eigenvalues of the Jacobian $D G$ are

$$
\mu_{1}(x)=c \quad \text { and } \quad \mu_{2}(x)=c(1-\varepsilon-\delta) .
$$

The eigenvectors are $v_{1}=(1,1)$ and $v_{2}=(-\delta, \varepsilon)$. The correspondent Lyapunov exponents are

$$
\lambda_{\|}=-a \ln a-(1-a) \ln (1-a) \text { and } \lambda_{\perp}=-a \ln a-(1-a) \ln (1-a)+\ln |1-d|
$$

with $d=\varepsilon+\delta$. Once again, the qualitative behavior of the system depends on the sum of the two coupling parameters and the parameter $d=\varepsilon+\delta$ has the role of the coupling parameter $\sigma$ refered in the network (5). We have a synchronization region $d_{1}<\varepsilon+\delta<d_{2}$, see Fig.5, instead of the synchronization interval.

See in Fig.4 the region where $\lambda_{\perp}<0$, in terms of the sum of the coupling parameters $d=\varepsilon+\delta \in[0,2]$ and for the function parameter $a \in[0.5,1]$. In particular for $a=0.8$, the graphic of $\lambda_{\perp}$, for the coupling version 2 of two tent maps, is presented at the right. We have $\lambda_{\perp}<0$, if $0.39<\varepsilon+\delta<1.61$.
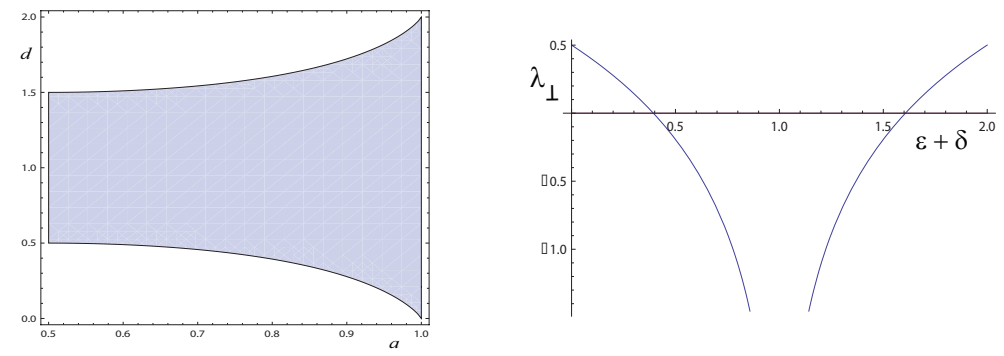

FIgURE 4 . In the left is the region where $\lambda_{\perp}<0$, in terms of the sum of the coupling parameters $d=\varepsilon+\delta \in[0,2]$ and for the function parameter $a \in[0.5,1]$. In the right is the graphic of $\lambda_{\perp}$, for the coupling version 2 of two tent maps, with $a=0.8$.

In Fig.5, we present the parameter synchronization region, $0.59<\varepsilon+\delta<1.87$. According to (9) and (10), in the particular case $n=1$, we evaluate the mutual information rate $I_{C}$ and the Kolmogorov-Sinai entropy $H_{K S}$. See in Fig.6 the graphics of $H_{K S}$ and $I_{C}$, for the coupling version 2 of two tent maps, with $a=0.8$. If $0.39<d=\varepsilon+\delta<1.61$ (complete synchronization), then $H_{K S}=I_{C}$. If $d<0.39$, then the MIR $I_{C}$ increases and $H_{K S}$ decreases. On the other hand, if $d>1.61$, then the MIR $I_{C}$ decreases and $H_{K S}$ increases. So, when there is no synchronization, errors may occur, while when there is complete synchronization the errors produced by the nonsynchronous trajectories should vanish and the channel transmits information in its full capacity.

\subsection{Complete network}

Consider a complete network with $\mathrm{N}$ nodes, see Fig.7, having in each node the same function, the skew-tent map (11). If, for instance, $N=4$, the adjacency $A$ and the Laplacian matrix $L$ are

$$
A=\left[\begin{array}{llll}
0 & 1 & 1 & 1 \\
1 & 0 & 1 & 1 \\
1 & 1 & 0 & 1 \\
1 & 1 & 1 & 0
\end{array}\right], \quad L=A-D=\left[\begin{array}{cccc}
-3 & 1 & 1 & 1 \\
1 & -3 & 1 & 1 \\
1 & 1 & -3 & 1 \\
1 & 1 & 1 & -3
\end{array}\right]
$$




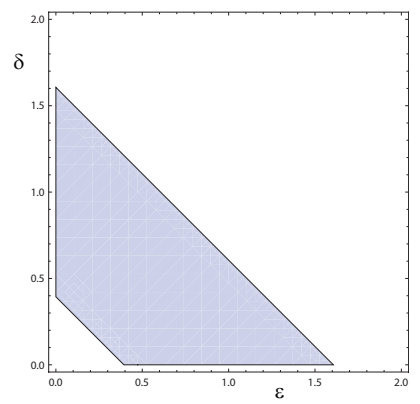

Figure 5. Parameter synchronization region, $0.59<\varepsilon+\delta<1.87$, for the coupling version 2 of two tent maps, with $a=0.8$.

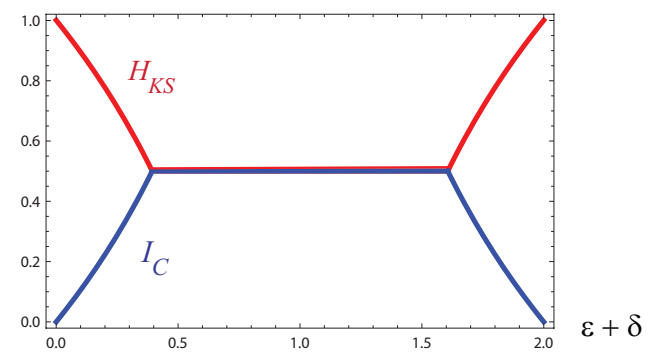

FiguRE 6. Graphic of $H_{K S}$ (in red) and $I_{C}$ (in blue), for the coupling version 2 of two tent maps, with $a=0.8$. If $0.39<d=\varepsilon+\delta<1.61$ (complete synchronization), then $H_{K S}=I_{C}$.

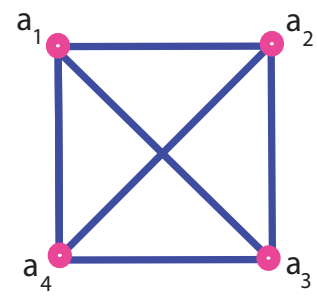

FiguRe 7. Complete network with 4 nodes.

This network is defined by the next system

$$
\left\{\begin{array}{l}
\dot{x}_{1}=f\left(x_{1}\right)+\sigma\left(-3 x_{1}+x_{2}+x_{3}+x_{4}\right) \\
\dot{x}_{2}=f\left(x_{2}\right)+\sigma\left(x_{1}-3 x_{2}+x_{3}+x_{4}\right) \\
\dot{x}_{3}=f\left(x_{3}\right)+\sigma\left(x_{1}+x_{2}-3 x_{3}+x_{4}\right) \\
\dot{x}_{4}=f\left(x_{4}\right)+\sigma\left(x_{1}+x_{2}+x_{3}-3 x_{4}\right)
\end{array}\right.
$$

where $\sigma$ is the coupling parameter. The Jacobian is

$$
J=\left[\begin{array}{cccc}
c-3 \sigma & \sigma & \sigma & \sigma \\
\sigma & c-3 \sigma & \sigma & \sigma \\
\sigma & \sigma & c-3 \sigma & \sigma \\
\sigma & \sigma & \sigma & c-3 \sigma
\end{array}\right],
$$


being $c$ the slope of $f$, given by (13). The eigenvalues of the Jacobian are $\mu_{1}=c, \mu_{2}=\mu_{3}=\mu_{4}=c-4 \sigma$. The first eigenvector is $(1,1,1,1)$ and the others are $(-1,0,0,1),(-1,0,1,0),(-1,1,0,0)$. So,

$$
\lambda_{\|}=\int \ln \left|\mu_{1}\right|=\int_{0}^{a} \ln \frac{1}{a}+\int_{a}^{1} \ln \left|\frac{-1}{1-a}\right|=-a \ln a-(1-a) \ln (1-a)
$$

and there are 3 identical transversal Lyapunov exponents, which are equal to

$$
\lambda_{\perp}=a \ln \left|\frac{1}{a}-4 \sigma\right|+(1-a) \ln \left|-\frac{1}{1-a}-4 \sigma\right| .
$$

Then, the number $n$ referred in Theorem (3.1) is $n=1$.

In a complete network with four nodes, there are six identical communication channels. Any pair of different nodes produces the same change of information, see Fig.7. The region, in the parameter plane $(\sigma, a)$, where $\lambda_{\perp}<0$, with $0.5 \leq a<1$ and $0 \leq \sigma \leq 0.6$, is displayed in Fig.8. For instance, for the tent map parameter $a=0.7$, we have $\lambda_{\perp}<0$ if the coupling parameter $\sigma \in[0.22,0.48]$.

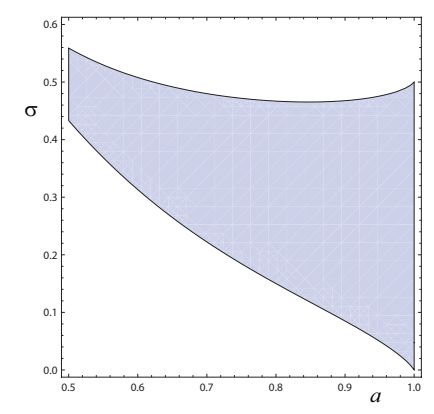

FiguRE 8. Region where $\lambda_{\perp}<0$, with $0.5 \leq a<1$ and $0 \leq \sigma \leq 0.6$.

If $\sigma=0$, there is no coupling, so no information is exchanged between any two nodes $\left(I_{C}=0\right)$. If $\sigma<0.22$, the larger is the coupling parameter $\sigma$, more synchronous become the system and more information is exchanged (larger $I_{C}$ ). The channel capacity for all 6 communication channels is achieved for $0.22<\sigma<0.48$, when $I_{C}=0.61=\min \left\{H_{K S}\right\}$ and the network become completely synchronized $\left(\lambda_{\perp}<0\right)$. On the other hand, if $\sigma>0.48$, then the MIR $I_{C}$ decreases and $H_{K S}$ increases. The graphics of the MIR $I_{C}$ and $H_{K S}$ are presented in Fig.9. The main results, Theorem (3.1), are verified.

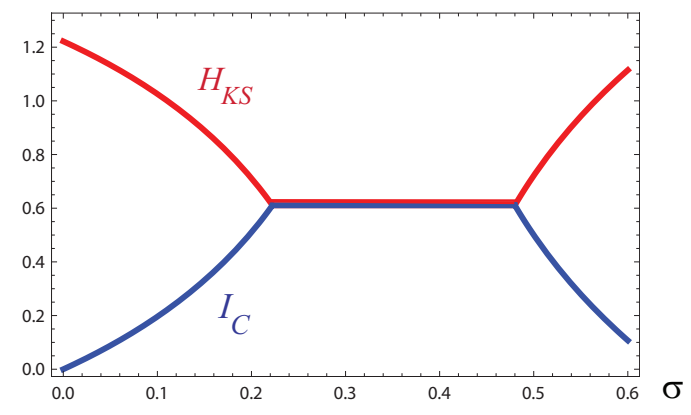

Figure 9. Graphic of $H_{K S}$ (in red) and $I_{C}$ (in blue), for a complete network with four nodes. 


\subsection{Lattice}

Consider a regular ring lattice, also called a nearest-neighbor coupling, a graph with $N$ nodes each one connected to $k$ neighbors, $\frac{k}{2}$ on each side. A lattice with an even number $N$ of elements and $K=2$ possess $\frac{N}{2}-1$ degenerate eigenvalues, and therefore $N-\frac{N}{2}+1$ distinct eigenvalues. There are only $N-\frac{N}{2}$ different transversal Lyapunov exponents and $N-\frac{N}{2}$ different minimal path lengths connecting the elements of the network. In this case, $I_{C}$ assumes only $N-\frac{N}{2}$ different values. It is reasonable to state that each different value corresponds to the exchange of information between elements, that have the same minimal path length.

We will consider a lattice having in each node the same function, the skew-tent map (11). If, for instance, $N=4$ and $K=2$, see Fig.10, we will have 2 different minimal path lengths, 2 different transversal Lyapunov exponents, i.e., the $n$ referred in Theorem (3.1), takes the value 2. The adjacency $A$ and the Laplacian matrix $L$ are

$$
A=\left[\begin{array}{llll}
0 & 1 & 0 & 1 \\
1 & 0 & 1 & 0 \\
0 & 1 & 0 & 1 \\
1 & 0 & 1 & 0
\end{array}\right], \quad L=A-D=\left[\begin{array}{cccc}
-2 & 1 & 0 & 1 \\
1 & -2 & 1 & 0 \\
0 & 1 & -2 & 1 \\
1 & 0 & 1 & -2
\end{array}\right]
$$

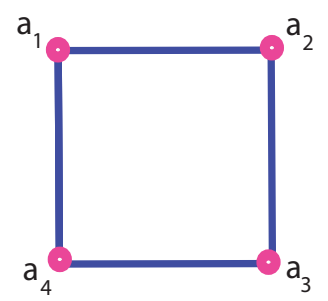

Figure 10. Lattice with $N=4$ and $K=2$.

This network is defined by the system

$$
\left\{\begin{array}{l}
\dot{x}_{1}=f\left(x_{1}\right)+\sigma\left(-2 x_{1}+x_{2}+x_{4}\right) \\
\dot{x}_{2}=f\left(x_{2}\right)+\sigma\left(x_{1}-2 x_{2}+x_{3}\right) \\
\dot{x}_{3}=f\left(x_{3}\right)+\sigma\left(x_{2}-2 x_{3}+x_{4}\right) \\
\dot{x}_{4}=f\left(x_{4}\right)+\sigma\left(x_{1}+x_{3}-2 x_{4}\right)
\end{array}\right.
$$

where $\sigma$ is the coupling parameter. The Jacobian is

$$
J=\left[\begin{array}{cccc}
c-2 \sigma & \sigma & 0 & \sigma \\
\sigma & c-2 \sigma & \sigma & 0 \\
0 & \sigma & c-2 \sigma & \sigma \\
\sigma & 0 & \sigma & c-2 \sigma
\end{array}\right]
$$

being $c$ the slope of $f$, given by (13). The eigenvalues of the Jacobian are $\mu_{1}=c, \mu_{2}=c-4 \sigma$ and $\mu_{3}=$ $\mu_{4}=c-2 \sigma$. The first eigenvector is $(1,1,1,1)$ and the others are $(-1,1,-1,1),(0,-1,0,1)$ and $(-1,0,1,0)$, respectively. Therefore,

$$
\lambda_{\|}=-a \ln a-(1-a) \ln (1-a)
$$

and there are two different transversal Lyapunov exponents

$$
\lambda_{\perp_{1}}=a \ln \left|\frac{1}{a}-4 \sigma\right|+(1-a) \ln \left|-\frac{1}{1-a}-4 \sigma\right|
$$


and

$$
\lambda_{\perp_{2}}=a \ln \left|\frac{1}{a}-2 \sigma\right|+(1-a) \ln \left|-\frac{1}{1-a}-2 \sigma\right| .
$$

For $0.5 \leq a<1$ and $0 \leq \sigma \leq 0.7$ we get the regions presented in Fig.11. In the first two graphics are the regions where each one of the transversal Lyapunov exponents is negative, but to have synchronization one must have all transversal Lyapunov exponents negative, so the region of synchronization, $\lambda_{\perp_{1}}<0$ and $\lambda_{\perp_{2}}<0$, is shown in the third graphic. For instance, for $a=0.8$, we get synchronization if $0.3<\sigma<0.47$. For $a=0.8$, the graphic of $H_{K S}$, given by Eq.(10), is displayed in Fig.12. In this lattice, with 4 nodes, there are two possible minimal path lengths. So, one may consider the MIR $I_{C_{1}}$ for those nodes that are one connection apart and the MIR $I_{C_{2}}$ for those nodes that are two connection apart, see Fig.13.
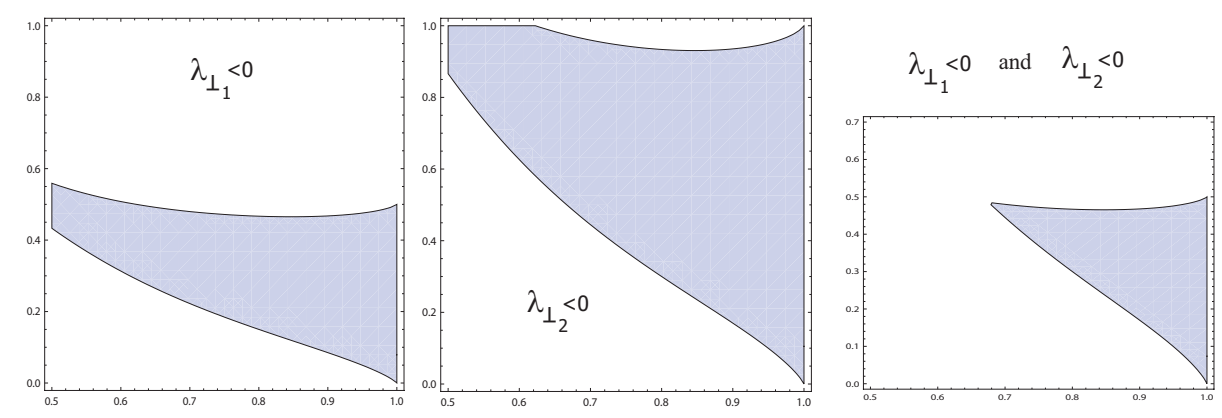

FIGURE 11. Regions where the transversal Lyapunov exponents are negative. In the vertical axis is the coupling parameter $\sigma$ and in the horizontal axis is the tent map parameter $a$.

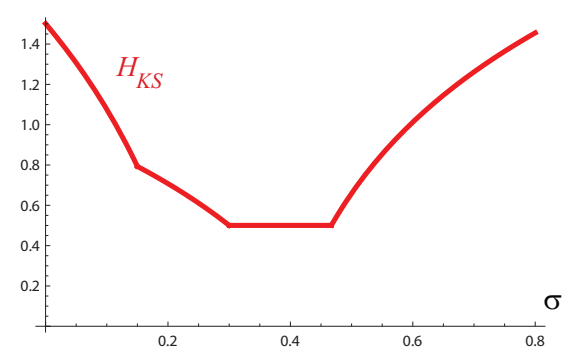

FIgURE 12. Graphic of $H_{K S}$ for the lattice with four nodes and $a=0.8$, as a function of the coupling parameter $\sigma$.
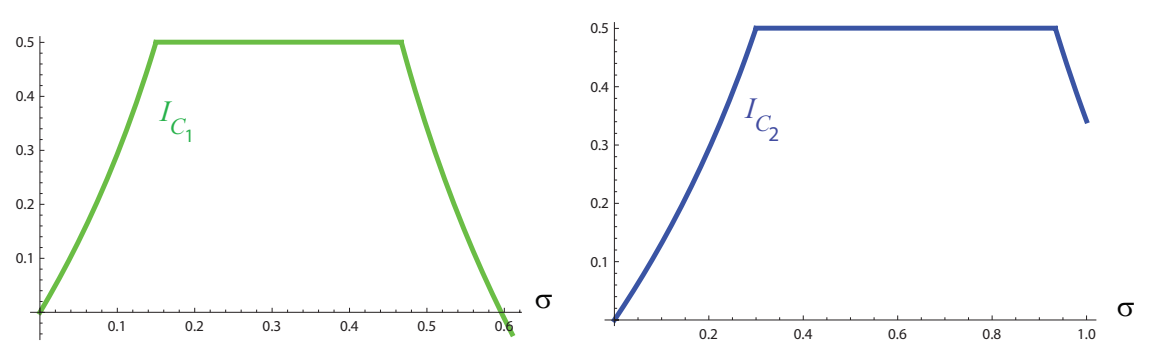

FIGURE 13. Graphics of $I_{C_{1}}$ and $I_{C_{2}}$, as functions of the coupling parameter $\sigma$, for the lattice with four nodes and $a=0.8$. 


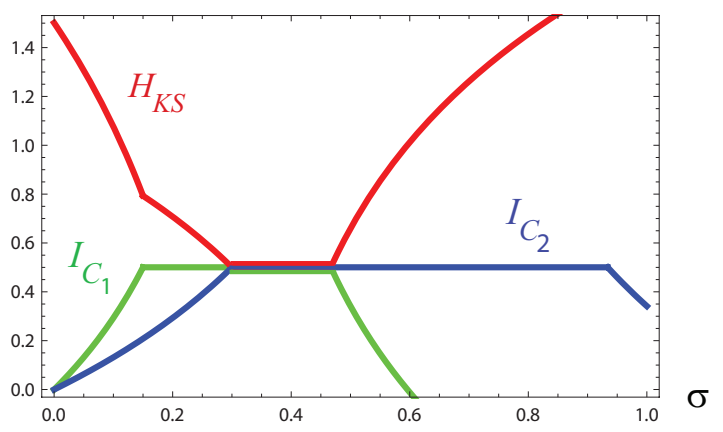

Figure 14. Graphic of $H_{K S}$ and $I_{C}$ for a lattice with $N=4$ nodes, having each one $K=2$ neighbors.

In Fig.14 we have the two mutual information rate $I_{C_{1}}, I_{C_{2}}$ and the Kolmogorov-Sinai entropy $H_{K S}$, as functions of the coupling parameter $\sigma$. In the interval of complete synchronization, $0.3<\sigma<0.47$, we have $I_{C_{1}}=I_{C_{2}}=H_{K S}$. For $\sigma<0.3$, the MIR $I_{C}$ increases more slowly for those nodes that are two connection apart $I_{C_{2}}$, than for those nodes that are one connection apart $I_{C_{1}}$. On the other hand, for $\sigma>0.47$, the mutual information rate decreases more slowly for those nodes that are one connection apart $I_{C_{1}}$, than for those nodes that are two connection apart $I_{C_{2}}$. Therefore, the results of Theorem (3.1) are verified.

\section{Conclusions}

We have studied the relationship between the level of synchronization and the amount of information that flows in a network. It was already known that as the coupling parameter increases from zero, the level of synchronization increases until a maximum and then decreases. For values of the coupling parameter in the so called synchronization interval, the network is in complete synchronization. Using the concept of mutual information rate, to measure of the information flux, and Lyapunov exponents, we proved that the more synchronized is the network, the larger is the information flux. In fact, the mutual information rate increases to a maximum at the synchronization interval and then decreases. Moreover, the Kolmogorov-Sinai entropy decreases until reaching a minimum at the synchronization interval and then increases. Some particular examples are given. We consider two different versions of coupling two maps, a complete network and a lattice, all of them having in the nodes identical skew-tent maps. Evaluating the transversal Lyapunov exponents, the mutual information rate of all communications channels and the Kolmogorov-Sinai entropy, we have confirmed our previous results.

\section{ACKNOWLEDGMENT}

This work is funded by FCT through the National Funds - Foundation for Science and Technology within the project PEst-OE/MAT/UI0006/2011, CIMA-UE, CEAUL and ISEL.

\section{REFERENCES}

[1] M. S. Baptista and J. Kurths, Chaotic channel, Phys. Rev. E, 72, 045202R, (2005).

[2] M. S. Baptista and J. Kurths, Information transmission in active networks, Phys. Rev. E, 77, 026205, (2008).

[3] M. S. Baptista, F. M. Moukam Kakmeni and C. Grebogi, Combined effect of chemical and electrical synapses in HindmarshRose neural networks on synchronization and the rate of information, Phys. Rev. E, 82, 036203, (2010).

[4] S. Boccaletti, J. Kurths, G. Osipov, D. L. Valladares and C. S. Zhou, The synchronization of chaotic systems, Physics Reports, 366, (2002), 1-101.

[5] A. Caneco, S. Fernandes, C. Gracio and J. L. Rocha, Networks synchronizability, local dynamics and some graph invariants, Dynamics, Games and Science I, Springer Proceedings in Mathematics, 1, (2011), 221-238.

[6] J. Cao and J. Lu, Adaptive synchronization of neural networks with or without time-varying delays, Chaos 16, 013133, (2006). 
[7] M. Hasler, Y. L. Maistrenko, An Introduction to the Synchronization of Chaotic Systems: Coupled Skew Tent Maps, IEEE Trans. Circuits Syst. I: Fundamental Theory and Applications, 44, 10, (1987), 856-866.

[8] A. N. Kolmogorov, A new metric invariant of transient dynamical systems and automorphisms in Lebesgue spaces, Dokl. Akad. Nauk SSSR, 119 (1958), 861-864.

[9] A. N. Kolmogorov, Entropy per unit time as a metric invariant of automorphisms, Dokl. Akad. Nauk SSSR, 124 (1959), 754-755.

[10] L. M. Pecora and T. L. Carroll, Synchronization in chaotic systems, Phys. Rev. Lett., 64, (1990), 821-824.

[11] L. M. Pecora and T. L. Carroll, Driving systems with chaotic signals, Phys. Rev. A, 44, (1991), 2374-2383.

[12] Ya. B. Pesin, Characteristic Lyapunov exponents and smooth ergodic theory, Russ. Math. Surv., 32, 4, (1977), 55-114.

[13] C. E. Shannon, A mathematical theory of communication, Bell System Technical J., 27, 379, (1948), 379-423.

[14] J. W, Shuai, K. W. Wong and L. M. Cheng, Synchronization of spatiotemporal chaos with positive conditional Lyapunov exponents, Phys Rev E, 56, 2272, (1997).

[15] F. Verhulst, Nonlinear Differential Equations and Dynamical Systems, Springer, Berlin, Heidelberg, (1990). 